\title{
AEOLUS L2 ACTIVITIES AT KNMI
}

\author{
D. P. Donovan*, Gert-Jan Marseille, Jos de Kloe and Ad Stoffelen.
}

Royal Netherlands Meteorological Institute (KNMI), PO Box 201, 3730 AE de Bilt, the Netherlands

*Email:Donovan@knmi.nl

\begin{abstract}
KNMI has had a long involvement with the recently launched Aeolus mission. Aeolus is an ESA Earth-Explorer mission and embarks the Atmospheric Laser Doppler Instrument (ALADIN). ALADIN is the first space-borne Doppler Wind Lidar (DWL) and was successfully launched in late August 2018. KNMI has had a long association with this mission and is sharing the software development of the L2B processor used to generate L2B wind products at ECMWF, develops related scene classification algorithms and performs calibration/validation activities. In addition, KNMI conducts more fundamental work for improving the retrieval of atmosphere optical properties, taking into account multiple scattering effects.
\end{abstract}

\section{INTRODUCTION}

ALADIN is a high spectral resolution Doppler lidar operating at $355 \mathrm{~nm}$ mounted on the Aeolus satellite. Aeolus orbits in a sun-synchronous dusk/dawn orbit at $320 \mathrm{~km}$ height. ALADIN currently emits 40-60 mJ pulses of circularly polarized light, slightly less than the opted $80 \mathrm{~mJ}$, at a PRF of $50 \mathrm{~Hz}$. The backscattered light is collected using a $1.5 \mathrm{~m}$ diameter telescope. The return signal is separated into two channels; the so-called "Mie" and "Rayleigh" channels.

The Mie channel uses a Fizeau interferometer coupled to an ACCD array to spectrally resolve and enable the separation of the spectrally narrow elastic backscatter (Mie) peak from clouds/aerosol and the spectrally broader return from atmospheric molecules. By measuring the spectral position of the Mie peak, the Doppler shift relative to the emitted signal frequency can be estimated and thus the line-of-sight velocity of the cloud/aerosol particles deduced. About $20 \%$ of the atmospheric samples have, in general, a cloud/aerosol return of sufficient signal-to-noise ratio to retrieve accurate winds, depending on the vertical sampling strategy.

The Rayleigh channel is built around a dual Fabry-Pérot interferometer. The etalon is split into two zones, which are imaged separately on to an ACCD detector. The two resulting subchannels are offset spectrally either side of the center frequency such that the spectrally broad Doppler shift of the atmospheric backscatter can be deduced using the ratio of the two branches of the Rayleigh channel returns. For more information see [1]. The Rayleigh channel is unique in that it provides wind information in clear air and it provides generally winds for about $80 \%$ of the samples, again depending on vertical sampling settings.

ALADIN is tilted 35 degrees off nadir in the direction perpendicular to the orbit track enabling the estimation of horizontally-projected line-ofsight (HLOS) atmospheric wind vector components with a typical resolution of $87 \mathrm{~km}$ along-track and a targeted accuracy of 2 to $3 \mathrm{~m} / \mathrm{s}$ throughout the troposphere and lower stratosphere. The vertical resolution is fixed to 24 bins in each channel with bin sizes equal to multiples of $250 \mathrm{~m}$, typically ranging from $250 \mathrm{~m}$ in the lowest $\mathrm{km}$ to 1 to $2 \mathrm{~km}$ up to an altitude of about $30 \mathrm{~km}$ for the Rayleigh channel.

The line-of-sight wind profiles (which are being assimilated into numerical weather prediction models and used to monitor atmospheric dynamics and circulation) are the primary Aeolus products. However, cloud and aerosol optical properties are also being produced with a horizontal resolution down to $3 \mathrm{~km}$, the basic Aeolus horizontal integration length, as secondary product. 


\section{KNMI ACTIVITIES}

As part of the ESA Aeolus DISC project, KNMI is leading the development of the so-called chainof-processors, an end-to-end cal/val and Aeolus product $\mathrm{R} \& \mathrm{D}$ tool, operated in an internet cloud and shared within the Aeolus Cal/Val team. It is used for end-to-end sensitivity tests in the wind processing chain and accumulates new calibration settings, algorithm and tool development and product monitoring and evolution. This tool is relevant for all scientific activities towards Aeolus product improvement.

\subsection{Cal/Val}

To support $\mathrm{Cal} / \mathrm{Val}$ activities of wind products, ECMWF and KNMI are involved in monitoring and diagnosing the optical properties and wind products from Aeolus, using prior weather information from the ECMWF model as reference. These fields are available at all Aeolus measurement locations and their availability facilitated a swift identification of anomalies in the Aeolus data processing, the resolution of some major anomalies and the beneficial assimilation of early and preliminary Aeolus data in the ECMWF model.

In addition to model data, ground-based data is used for validation. In fact, KNMI combines all data types in triple collocation for error attribution. In particular, over land Europe sees a large volume of tropospheric wind data emerging through Mode-S airplane winds [2]. These winds are collocated with Aeolus and ECMWF winds for error analysis at each vertical level from the ground to $11 \mathrm{~km}$.

Finally, KNMI launches radiosondes from Paramaribo in Suriname in order to obtain highresolution tropical wind, humidity and temperature profiles collocated with Aeolus overpasses. Elsewhere, this is augmented with other in-situ continuous profiling networks, such as Cabauw in the Netherlands.

\subsection{Scene classification and optical properties}

Scene classification is an important component of the Level-2B wind processor (L2Bp). Its main objective is to detect cloud and aerosol layers along the Aeolus track. The result is a binary map, also denoted feature mask, used in the L2Bp classification procedure as input for the accumulation of Mie and Rayleigh signals from measurement ( $2.88 \mathrm{~km}$ along track) to observation level (87 km along track).

Two options for scene classification have been implemented in the L2Bp, (i) based on the application of a threshold value to the scattering ratio estimate calculated by the Level-1B processor and (ii) from the Optical Properties Code (OPC), which is part of the L2Bp. Both options are operated on measurement level, which poses a challenge because of the high levels of measurement noise on top of the atmospheric return signal.

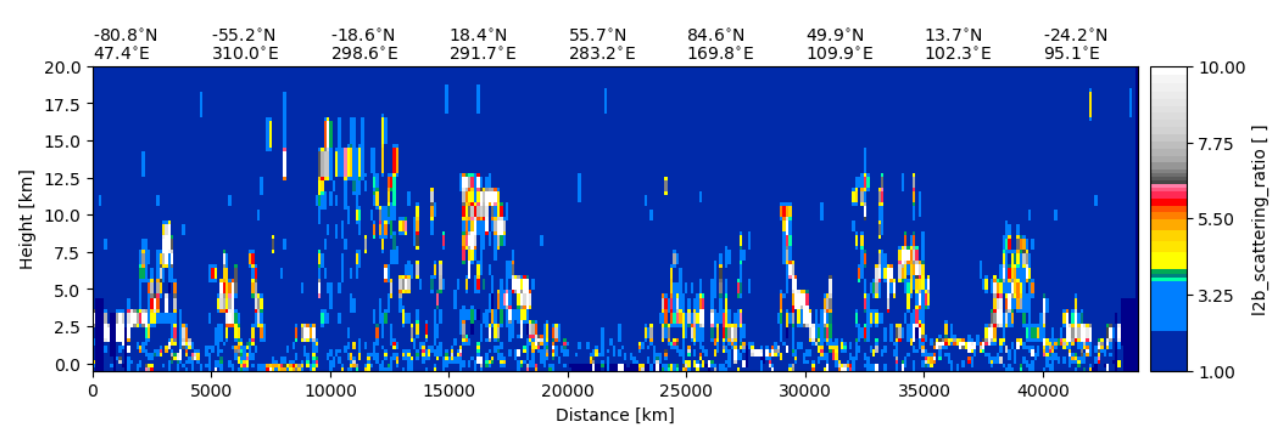

Figure 1: Sample preliminary L2B scattering ratios estimates (derived from Rayleigh channel)

Sample L2B scattering rato preliminary results are shown in Figure 1. Both L2B and L1B options are complementary because the L1B scattering ratio is inferred from the Mie channel signal only, while OPC uses the Rayleigh channel signal only. OPC also contributes to a mission risk reduction in case the Mie channel performs less than anticipated. Since scene classification is a crucial procedure in the $\mathrm{L} 2 \mathrm{Bp}$, the quality of produced winds strongly relies on the performance of the classification procedure in case of cloud and/or aerosol presence.

The L2 processor development in the ESA DISC project and in collaboration with ECMWF is 
being further optimized for to optimize the NWP impact.

\subsection{Impact of Lidar Multiple Scattering on ALADIN retrievals.}

For much of the ALADIN processing it is implicitly assumed that lidar multiple scattering effects are negligible. It is true that the receiver field-of-view (fov) of Aeolus is small (0.019) mrads), a value which is an order of magnitude smaller than those commonly used by terrestrial lidar systems. On this basis it may be supposed that lidar multiple-scattering effects present in Aeolus return signals will be small and can safely be ignored. This assumption, however, is not always valid.
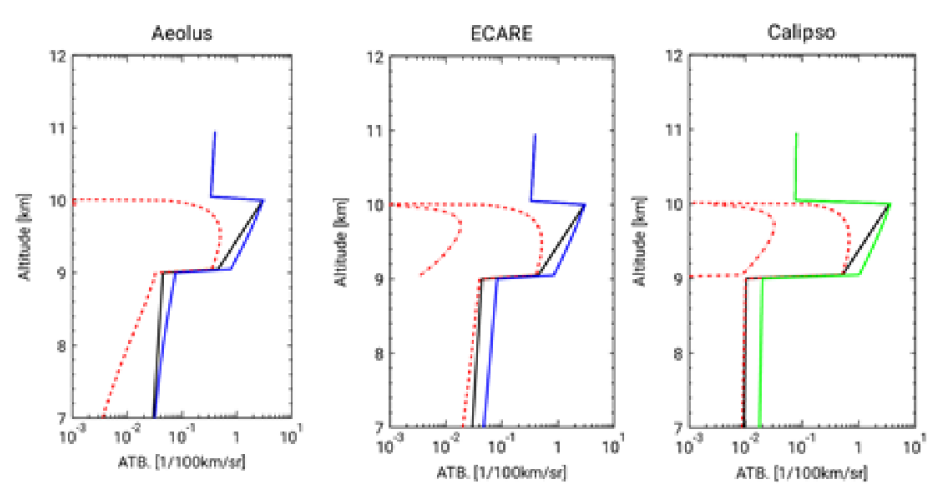

Figure 2: Simulated relative (total Ray+Mie) attenuated backscatter profiles corresponding to an idealized cirrus cloud with an optical thickness of 1 and an effective particle size of 20 microns. The Black lines correspond to the single-scatter only return, the Red lines show the 2nd and 3rd order scattering respectively ( $3 \mathrm{rd}$ order return for the Aeolus case is too small to be shown) and the Blue and Green lines represent the total single scattering $(\mathrm{SS})+$ multiple scattering (MS) returns.

The relative strength of Multiple-Scattering (MS) in lidar returns depends on the laser divergence, the receiver field-of-view (fov), the distance from the target, the target extinction coefficient and the scattering phase function of the target. Of particular importance is the relative strength of the forward-scattering lobe of the phase function which increases with increasing particle sizes. The more forward scattering that occurs, the more chance multiple-scattering photons will stay within the receiver fov. This can be relevant even when the optical thickness of the target is small. This means that, e.g., cirrus clouds whose typical particle effective radii can be several -tens of microns are general targets where MS effects are particularly evident. Figure 1 shows an example of the results of an approximate analytical model [3] to an idealized cirrus cloud of optical thickness 1.0 with an effective particle size of 20 microns. Cases corresponding to CALIPSO, ATLID (the EarthCARE lidar) and Aeolus are shown. Here it can be seen that within the cloud the MS contribution can approach (and in the case of CALIPSO) exceed that of the SS return. Below the cloud, the return from the molecular Rayleigh atmosphere also contains a significant MS component arising from forward scattering events within the cloud above. In the case of CALIPSO a slight decay in the below-cloud MS return is present. For ATLID, the decay is somewhat more apparent and is obvious for Aeolus.
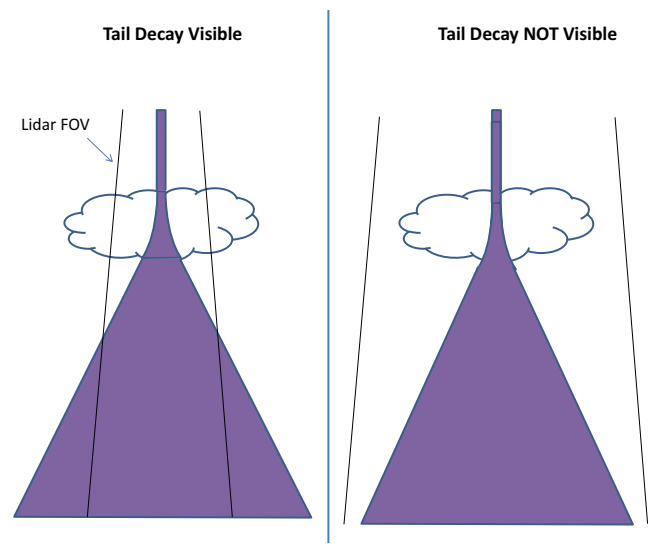

Figure 3: Schematic illustrating the basic mechanism behind the appearance of below cloud "tails" in spacebased lidar returns.

The mechanism behind the appearance of these tails is illustrated schematically in Figure 2. Basically the scattering events occurring within the cloud serve to broaden the angular-spatial extend of the lidar beam. This beam then emerges from the cloud-base with a certain effective diameter and divergence. If the effective divergence is small compared to the lidar fov then no decay will be visible, but if this is not the case, then these type of "tails" will be apparent. Such tails indeed can be observed for CALIPSO but only for cases of relatively small cirrus particles (e.g. see Appendix A of [4]).

The approach used in [3] is limited in its accuracy and cannot accurately address the effects of MS on the spectral-polarization state of the lidar 
return. However, accurate lidar Monte-Carlo calculations can be used for this purpose. In this work the lidar radiative transfer model and instrument model of the EarthCARE simulator will be applied to the case of Aeolus. As well as exactly treating the lidar multiple-scattering also calculates the spectral-polarization state of the return signal $[4,5]$.

\section{Implications for Aeolus}

Neglecting lidar MS effects in the retrieval algorithms may lead to a number of issues in the L2 products if not ameliorated in some manner.

The retrieval of cloud optical depth will be underestimated by up to several tens of percent if MS effects are not accounted for. For example, if the method of comparing the directly above and below cloud Rayleigh returns would be applied to the case shown in Figure 1 then the cloud transmission would be off by a factor of more than 2.

The polarization-spectral state of the MS return will not be identical to that of the SS only return. In particular, the greater angular distribution of the MS returns would be expected to alter the spectra of the in-cloud and even below cloud returns. The impact on the spectra shape are expected to be small though due to the small laser divergence and receiver field-of-view and not important for wind-retrievals.

In order to facilitate the investigation of anomalies and the possible effects of multiple-scattering on Aeolus products the EarthCARE simulator lidar modules [5] is being extended and tested in order to accurately handle Aeolus parameters and viewing geometry. This investigation contributes to the broader assessment of the feature detection/classification and optical properties retrievals with a particular effort directed to the quantification of multiple-scattering effects on the instrument performance (e.g., calibration, crosstalk) and on the feature detection/classification and optical properties and wind retrievals.

\section{SUMMARY}

KNMI is supporting the Aeolus mission in several areas. The activities at KNMI include leading the development of the so-called chain-of-processors,, participating in data monitoring and various
$\mathrm{Cal} / \mathrm{Val}$ activities and developing the L2B Scene classification algorithm (an important component of the Level-2B wind processor). In addition, more fundamental research relevant for Aeolus (e.g. the impact of MS on Aeolus signals) is also be conducted.

\section{ACKNOWLEDGEMENTS}

This work is supported in by ESA under the Aeolus DISC contract no. 40000126336/18/I-BG.

\section{REFERENCES}

[1] "ADM-Aeolus (Atmospheric Dynamics Mission)". https://directory.eoportal.org/web/eoportal/satellitemissions/a/aeolus ESA. Retrieved 18 Feb, 2019.

[2] Haan, S. de, and L. J. Bailey, and J. E. Konnen, 2013: Quality assessment of Automatic Dependent Surveillance Contract (ADS-C) wind and temperature observations from commercial aircraft, Atmos. Meas. Tech., 6, $199-206$.

[3] E.W. Eloranta, 1998: Practical model for the calculation of multiply scattered lidar returns, Appl. Opt. 37, 2464-2472 (1998).

[4] Reverdy, M., H. Chepfer, D. Donovan, V. Noel, G. Cesana, C. Hoareau, M. Chiriaco, and S. Bastin , 2015: An EarthCARE/ATLID simulator to evaluate cloud description in climate models, J. Geophys. Res. Atmos., 120, 11,090-11,113.

[5] Donovan, D. P., Klein Baltink, H., Henzing, J. S., de Roode, S. R., and Siebesma, A. P. 2015: A depolarisation lidar-based method for the determination of liquid-cloud microphysical properties, Atmos. Meas. Tech., 8, 237-266. 